\title{
Relationship between Behavioral Lifestyle and Mental Health Status Evaluated Using the GHQ-28 and SDS Questionnaires in Japanese Factory Workers
}

\author{
Miyuki SUDA, Kunio NAKAYAMA and Kanehisa MORIMOTO* \\ Department of Social and Environmental Medicine, Osaka University Graduate School of Medicine, Osaka \\ University, Suita, Osaka 565-0871, Japan
}

Received November 18, 2004 and accepted March 10, 2007

\begin{abstract}
In order to clarify the relationships between lifestyle behaviors and mental health status in Japanese workers, the 28-item General Health Questionnaire (GHQ-28) and Zung's Self-rating Depression Scale (SDS) were administered to 254 male factory workers; further, eight of their personal health practices were surveyed using the Health Practice Index (HPI). Few studies have used the GHQ-28 and SDS simultaneously; moreover, to date, no study has included the lifestyle factors in these scales. We used these tools for the concurrent assessment of mental health status and classified the subjects into high-risk and low-risk groups. The GHQ-28 and SDS scores correlated negatively with the HPI scores. In the older age group, these scores decreased significantly corresponding to an increase in the total number of favorable lifestyle practices followed. Significant relationships were observed between the GHQ-28 subscales and the HPI. Multiple logistic regression analysis revealed that four of the eight health practices surveyed were significantly related to the mental health status in all subjects. Thus, good health practices appear to be closely associated with good mental health status. However, to establish our results, larger study populations are required for further studies in various occupational fields.
\end{abstract}

Key words: Lifestyle, Health practice, Mental health, Stress, Depression, HPI, GHQ-28, SDS

\section{Introduction}

Lifestyle factors such as smoking, alcohol consumption, and physical activities are known to be associated with morbidity and mortality from cancers ${ }^{1-5}$. We have observed that unhealthy lifestyles cause not only chromosomal damage ${ }^{6}$ but also a decrease in natural killer cell activities ${ }^{7}$. Furthermore, we have demonstrated that health attitudes are closely related to the overall lifestyle, which was assessed in a Japanese community through eight health factors, namely, cigarette smoking, alcohol consumption, breakfast consumption, sleeping hours, working hours, physical exercise, nutritional balance, and mental stress ${ }^{8)}$. The collective evaluation of these health factors constitutes the

*To whom correspondence should be addressed.
Health Practice Index (HPI).

Today, with changes in the industrial structure, the mental as well as physical health status of workers has received considerable attention. The General Health Questionnaire $(\mathrm{GHQ})^{9)}$ is a 60 -item self-administered screening tool designed to detect nonpsychotic psychiatric illnesses. The GHQ-28, a shorter version of the original GHQ, has been shown to be reliable and valid ${ }^{10-12}$. We have reported that lifestyles affect mental health; moreover, strong negative correlations exist between the total number of favorable lifestyle practices as indicated by the HPI and the scores on the GHQ-28 and its four subscales (somatic symptoms, anxiety-insomnia, social dysfunction, and severe depression) ${ }^{13}$. Moreover, we have observed that several constituents of the HPI-mental stress, nutritional balance, physical exercise, and sleeping hours-were particularly 
associated with the total and subscale GHQ-28 $\operatorname{scores}^{13)}$. Furthermore, the validity of Zung's Self-rating Depression Scale (SDS) has been demonstrated in several studies ${ }^{14,15)}$. This test is sensitive enough to detect depressive symptoms in normal populations ${ }^{16}$. Its Japanese version ${ }^{17,18)}$ has been ascertained to possess good internal consistency and testretest reliability, and has been used in many research projects such as population surveys ${ }^{16}$. In addition, it has been shown that a number of favorable lifestyle practices, including working hours, are associated with the SDS scores ${ }^{19,20)}$. However, in Japan, modern workplaces are changing constantly, with the adoption of new working conditions (e.g., the introduction of the flextime system, the implementation of five-day work week, and the allowance of child care leave). Therefore, these older studies ${ }^{10,19,20)}$ are outdated to draw meaningful conclusions.

Since the severe depression subscale of the GHQ-28 is not suitable for assessing mild depression, the use of both the GHQ-28 and SDS can enable to assess a variety of aspects of the mental health status in a normal population. However, to date, only a few studies have used these scales simultaneously ${ }^{21,22}$. In bereaved subjects, the psychological distress assessed by the GHQ-28 and SDS decreased significantly over the duration of the study ${ }^{21)}$. The GHQ-28 and SDS were also used by Toda et al. ${ }^{22)}$ to assess stress reactions. Subjects obtaining high scores on the GHQ-28 and SDS are thought to suffer from severe psychological distress. Moreover, both the 30-item version of the GHQ, i.e., the GHQ-30, and SDS adequately identified cases of minor mental disorders and depressive disorders in early pregnancy ${ }^{23)}$. In a study that used the Structured Clinical Interview for DSMIII-R Personality Questionnaire (SCID-II Screen), SDS, and GHQ-30 to assess 781 male engineers and 214 male clerical workers at a computer manufacturing factory, it was observed that the scores for borderline and dependent personality traits were significantly lower in the engineers than in the clerical workers who were high school graduates ${ }^{2}$. These studies focused mainly on the various aspects of mental health status alone; further, to our knowledge, no research has been conducted regarding the effects of lifestyle factors on mental health. Moreover, only two studies were conducted on normal populations, while most of the other studies focused on clinical populations $^{2,22)}$.

Therefore, in the present study, in order to determine the relationships between lifestyle behaviors and the major scales used to assess mental health, we administered questionnaires to a group of male factory workers in Osaka, Japan. Lifestyle factors were evaluated using the HPI, while the mental health status was evaluated using the GHQ- 28 and SDS. The subjects were classified into two age groups, i.e., $\leq 39 \mathrm{yr}$ and $\geq 40 \mathrm{yr}$, similar to our previous study ${ }^{13)}$; further, the results of this study were compared with those of previous studies.

\section{Subjects and Methods}

\section{Subjects}

In October 2003, 344 employees from a factory in Osaka producing metal-cutting tooling and high impact custom cemented carbide were surveyed using self-administered questionnaires. The questionnaires were completed by 254 male workers (i.e., the valid response rate was $73.8 \%$ ). The mean age $( \pm \mathrm{SD})$ of these workers was $42.7( \pm 10.9) \mathrm{yr}$. This study was approved by the Ethics Committee of the Osaka University Graduate School of Medicine.

\section{Measurement of lifestyle behavior}

We used a self-administered questionnaire consisting of eight items for assessing health-related lifestyle practice according to the HPI, which was developed by Morimoto ${ }^{7,8)}$ and was based upon the studies conducted in the United States by Breslow and his colleagues ${ }^{3-5}$. The cultural differences were taken into consideration prior to the construction of this questionnaire. Points based on the HPI scoring system were assigned for positive responses to eight lifestyle items: smoking habits, drinking habits, daily breakfast consumption, appropriate duration of daily sleep, appropriate duration of daily work, regular physical activity, appropriate levels of subjective stress, and nutritionally balanced diet. Each item had two to six possible answers and was assigned a dichotomized value of 1 (good lifestyle) or 0 (poor lifestyle) according to the Morimoto's criteria. The scores for all items were added to generate the overall HPI score that ranged from 0 to 8 and provided a comprehensive evaluation of lifestyle behaviors. The subjects were classified into one of the three categories according to their HPI scores ${ }^{3,4)}$, namely, "poor" (HPI score, 0-4), "moderate" (HPI score, 5 or 6), and "good" (HPI score, 7 or 8 ).

\section{Measurement of mental health status}

The Japanese version of the GHQ-28 ${ }^{12)}$ and the SDS ${ }^{17,18)}$ were used to assess the mental health status. The GHQ-28 has four subscales, each consisting of seven items. The items were scored using a Likert-type scoring system, i.e., 1, 2, 3, and 4 , for the response categories; these scores were then converted into corresponding binary values of $0,0,1$, and 1. The sum of the scores can be interpreted as an indicator of the severity of psychological distress. The subjects were classified into higher and lower GHQ-28 groups based on a 
cutoff score of 8 .

The SDS consists of 20 items with four-point answer scales. The sum of the scores was categorized as "good" (SDS score <42), "mild depression" (SDS score $=42-48$ ), "moderate depression" (SDS score $=49-56$ ), and "severe depression" (SDS score $>57)^{14,20)}$. Subjects with SDS scores of 49 or more were included in the higher SDS group, while the remaining individuals were included in the lower SDS group.

Combining the scores from both scales, the subjects were categorized into four groups: (a) GHQ-28 score $\leq 7$ and SDS score $\leq 48$, (b) GHQ- 28 score $\geq 8$ and SDS score $\leq 48$, (c) GHQ-28 score $\leq 7$ and SDS score $\geq 49$, and (d) GHQ-28 score $\geq 7$ and SDS score $\geq 49$. Next, we separated the (a) and (d) groups; these were defined as the low-risk and high-risk groups, respectively, with regard to the mental health status.

\section{Data analysis}

Pearson's correlation coefficient was used to analyze the association between the mental health status (the GHQ28 and SDS scores) and the lifestyle factors (HPI scores) in both the age groups. A one-way analysis of variance (ANOVA) was performed for comparison among the four groups formed based on the mental health status (groups (a)-(d)) with respect to the HPI scores. In addition, by using one-way ANOVA, we determined the relationship between the GHQ-28 subscales and the HPI groups in order to compare the differences with the results of our previous studies $^{13,20)}$. Moreover, to identify the specific lifestyle factors that were significantly associated with mental health status after adequate control for the confounding factors, we performed three types of multiple logistic regression analysis; based on the mental health status, the binary value of 0 or 1 was assigned to the dependent variable when the subject belonged to (1) the lower or the higher GHQ-28 groups, (2) the lower or the higher SDS groups, and (3) the low-risk or the high-risk groups, respectively. Moreover, the GHQ-28 subscales were analyzed on the basis of our previous study ${ }^{13)}$; the binary value 0 or 1 was assigned to the dependent variable when the subject belonged to the group in which (1) somatic symptoms score was $\geq 2$ or $\leq 1$, (2) anxiety-insomnia score was $\geq 1$ or $\leq 0$, (3) social dysfunction score was $\geq 6$ or $\leq 5$, and (4) severe depression score was $\geq 1$ or $\leq 0$, respectively. The independent variables were age and the eight health practices; the "good" and "poor" practices were scored as 0 and 1, respectively. The analyses were performed using SPSS version 12.0J (SPSS Japan Institute Inc., Tokyo, Japan).
Table 1. Scores on GHQ-28, SDS, and HPI by age (yr): average \pm standard deviation

\begin{tabular}{lccc}
\hline \multicolumn{1}{c}{ Age } & GHQ-28 & SDS & HPI \\
\hline$\leq 39(\mathrm{n}=96)$ & $5.91 \pm 3.96$ & $40.33 \pm 8.25$ & $5.03 \pm 1.47$ \\
$\geq 40(\mathrm{n}=158)$ & $5.72 \pm 4.06$ & $40.61 \pm 7.46$ & $4.87 \pm 1.33$ \\
All $(\mathrm{n}=254)$ & $5.79 \pm 4.01$ & $40.50 \pm 7.75$ & $4.93 \pm 1.38$ \\
\hline
\end{tabular}

Table 2. Pearson's correlation coefficients among the scores of GHQ28, SDS, and HPI

\begin{tabular}{lcc}
\hline & Age & HPI \\
\hline GHQ-28 & $\leq 39$ & -0.177 \\
& $\geq 40$ & $-0.311^{*}$ \\
& All & $-0.256^{*}$ \\
\hline \multirow{2}{*}{ SDS } & $\leq 39$ & $-0.255^{*}$ \\
& $\geq 40$ & $-0.352^{*}$ \\
& All & $-0.311^{*}$ \\
\hline
\end{tabular}

${ }^{*} p<0.01$.

\section{Results}

The HPI, GHQ-28, and SDS scores for each age group are shown in Table 1. No significant differences were observed between the age groups for any of the scores (Student's $t$ test). Pearson's correlation coefficients between mental health and the lifestyle factors (Table 2) indicate that almost all values were significantly correlated with each other. The GHQ-28 and SDS scores were negatively correlated with the HPI scores.

The HPI scores were classified according to the mental health status and lifestyle factors (Table 3). In the older age group, a one-way ANOVA demonstrated a significant relationship between the mental health status and the HPI scores $(\mathrm{F}=5.127, p=0.002)$. The GHQ-28 scores for each age group are shown in Table 4 . In both the age groups, the total GHQ-28 score as well as the four subscale scores decreased with a corresponding increase in the total number of favorable lifestyle practices, as indicated by the HPI. In the older age group, significant relationships with the HPI scores were observed for anxiety-insomnia, social dysfunction, and the total GHQ-28 scores $(\mathrm{F}=5.966,4.879$, and 5.890; $p=0.003,0.009$, and 0.003, respectively).

The results of the multiple logistic regression analysis are shown in Table 5. Mental stress was significantly positively related to the total GHQ-28 scores $(p=0.001$, $95 \%$ Confidence Interval $(\mathrm{CI})=1.54-4.73)$, somatic symptoms ( $p=0.002,95 \% \mathrm{CI}=1.44-4.78)$, anxiety-insomnia 
Table 3. HPI scores by mental health status and lifestyles: average \pm standard deviation

\begin{tabular}{|c|c|c|c|c|c|c|c|c|c|c|}
\hline \multirow[t]{2}{*}{ Age } & \multirow[t]{2}{*}{ Mental health status } & & \multicolumn{8}{|c|}{ HPI groups } \\
\hline & & & Poor & $\mathrm{N}$ & Moderate & $\mathrm{N}$ & Good & $\mathrm{N}$ & Total & $\mathrm{N}$ \\
\hline \multicolumn{11}{|l|}{$\leq 39$} \\
\hline & $\mathrm{GHQ} \leq 7$ and $\mathrm{SDS} \leq 48$ & (Low-risk) & $3.25 \pm 0.72$ & 20 & $5.47 \pm 0.51$ & 32 & $7.18 \pm 0.40$ & 11 & $5.06 \pm 1.50$ & 63 \\
\hline & $\mathrm{GHQ} \geq 8$ and $\mathrm{SDS} \leq 48$ & & $3.60 \pm 0.89$ & 5 & $5.54 \pm 0.52$ & 13 & $7.50 \pm 0.71$ & 2 & $5.25 \pm 1.29$ & 20 \\
\hline & $\mathrm{GHQ} \leq 7$ and $\mathrm{SDS} \geq 49$ & & $3.50 \pm 0.71$ & 2 & $5.00 \pm 0.00$ & 1 & - & - & $4.00 \pm 1.00$ & 3 \\
\hline & $\mathrm{GHQ} \geq 8$ and $\mathrm{SDS} \geq 49$ & (High-risk) & $3.00 \pm 1.15$ & 4 & $5.60 \pm 0.55$ & 5 & $7.00 \pm 0.00$ & 1 & $4.70 \pm 1.70$ & 10 \\
\hline & Total & & $3.29 \pm 0.78$ & 31 & $5.49 \pm 0.50$ & 51 & $7.21 \pm 0.43$ & 14 & $5.03 \pm 1.47$ & 96 \\
\hline \multicolumn{11}{|l|}{$\geq 40^{*}$} \\
\hline & GHQ $\leq 7$ and $\mathrm{SDS} \leq 48$ & (Low-risk) & $3.65 \pm 0.61$ & 31 & $5.52 \pm 0.50$ & 54 & $7.21 \pm 0.43$ & 14 & $5.17 \pm 1.29$ & 99 \\
\hline & $\mathrm{GHQ} \geq 8$ and $\mathrm{SDS} \leq 48$ & & $3.24 \pm 0.75$ & 17 & $5.47 \pm 0.51$ & 17 & $7.00 \pm 0.00$ & 1 & $4.43 \pm 1.36$ & 35 \\
\hline & $\mathrm{GHQ} \leq 7$ and $\mathrm{SDS} \geq 49$ & & $3.67 \pm 0.58$ & 3 & - & - & - & - & $3.67 \pm 0.58$ & 3 \\
\hline & $\mathrm{GHQ} \geq 8$ and $\mathrm{SDS} \geq 49$ & (High-risk) & $3.58 \pm 0.67$ & 12 & $5.44 \pm 0.53$ & 9 & - & - & $4.38 \pm 1.12$ & 21 \\
\hline & Total & & $3.52 \pm 0.67$ & 63 & $5.50 \pm 0.50$ & 80 & $7.20 \pm 0.41$ & 15 & $4.87 \pm 1.33$ & 158 \\
\hline
\end{tabular}

$* p<0.01$.

Table 4. GHQ-28 subscale scores by age and lifestyles: average \pm standard deviation

\begin{tabular}{llcccc}
\hline \multirow{2}{*}{ Age } & GHQ-28 subscales & \multicolumn{4}{c}{ HPI groups } \\
& & Poor & Moderate & Good & Total \\
\hline$\leq 39$ & & & & \\
& & & & & \\
& Somatic symptoms & $1.45 \pm 1.09$ & $1.12 \pm 1.18$ & $0.64 \pm 0.84$ & $1.16 \pm 1.13$ \\
& Anxiety-Insomnia & $0.81 \pm 1.08$ & $0.73 \pm 1.22$ & $0.71 \pm 1.14$ & $0.75 \pm 1.15$ \\
& Social dysfunction & $4.19 \pm 2.15$ & $3.98 \pm 2.34$ & $2.64 \pm 2.41$ & $3.85 \pm 2.32$ \\
& Severe depression & $0.19 \pm 0.91$ & $0.14 \pm 0.49$ & $0.07 \pm 0.27$ & $0.15 \pm 0.63$ \\
& & & & \\
& Somatic symptoms & $1.30 \pm 1.24$ & $1.08 \pm 1.29$ & $0.53 \pm 0.83$ & $1.11 \pm 1.25$ \\
& Anxiety-Insomnia* & $1.10 \pm 1.52$ & $0.46 \pm 0.90$ & $0.33 \pm 0.62$ & $0.70 \pm 1.21$ \\
& Social dysfunction* & $4.22 \pm 2.24$ & $3.71 \pm 2.43$ & $2.13 \pm 2.26$ & $3.77 \pm 2.40$ \\
& Severe depression & $0.19 \pm 0.88$ & $0.08 \pm 0.47$ & $0.20 \pm 0.56$ & $0.13 \pm 0.67$ \\
& Total & $6.81 \pm 4.43$ & $5.33 \pm 3.67$ & $3.20 \pm 2.96$ & $5.72 \pm 4.06$ \\
\hline
\end{tabular}

$* p<0.01$.

$(p=0.000,95 \% \mathrm{CI}=2.03-6.16), \mathrm{SDS}(p=0.029,95 \% \mathrm{CI}$ $=1.09-5.29)$, and the high-risk vs. low-risk groups for mental health status ( $p=0.006,95 \% \mathrm{CI}=1.44-8.69)$. Nutritional balance was significantly negatively associated with the SDS scores $(p=0.000,95 \% \mathrm{CI}=2.65-16.16)$ and the high-risk vs. low-risk groups for mental health status ( $p=0.005,95 \%$ $\mathrm{CI}=1.59-13.91)$. Breakfast consumption was significantly negatively related to the total GHQ-28 scores $(p=0.022$, $95 \% \mathrm{CI}=1.13-4.84)$ and somatic symptoms $(p=0.000$, $95 \% \mathrm{CI}=2.12-9.69)$. Sleeping hours were significantly negatively associated with somatic symptoms $(p=0.041$, $95 \% \mathrm{CI}=1.03-4.15)$ and social dysfunction $(p=0.044$, $95 \% \mathrm{CI}=1.02-3.73)$.

\section{Discussion}

Our present study showed that a high total number of favorable lifestyle practices, as indicated by the HPI, had a negative relationship with the mental health status, which was measured by the GHQ-28 and SDS. Stronger relationships were observed in subjects in the older age group. These findings support the existence of a close relationship between the overall lifestyle and mental health status; this is consistent with the findings from previous studies ${ }^{24-26)}$. Simonsick found no association between health habits and mental health in young men ${ }^{25}$. However, we observed a correlation between the HPI and SDS scores in the younger age group. It has been suggested that modern adolescent depression is highly correlated with lifestyle behavior. The SDS measures only the depression status, whereas the GHQ- 
Table 5. Relationships of age and lifestyle to mental health: odds ratio for multiple logistic regression analysis ${ }^{\mathrm{a}}$

\begin{tabular}{|c|c|c|c|c|c|c|c|}
\hline \multirow[b]{3}{*}{ Independent variables } & \multicolumn{7}{|c|}{ Dependent variables } \\
\hline & \multicolumn{5}{|c|}{ GHQ-28 } & \multirow[t]{2}{*}{ SDS } & \multirow{2}{*}{$\begin{array}{l}\text { High risk vs. } \\
\text { Low risk }\end{array}$} \\
\hline & Total & $\begin{array}{c}\text { Somatic } \\
\text { symptoms }\end{array}$ & $\begin{array}{l}\text { Anxiety- } \\
\text { insomnia }\end{array}$ & $\begin{array}{c}\text { Social } \\
\text { dysfunction }\end{array}$ & $\begin{array}{c}\text { Severe } \\
\text { depression }\end{array}$ & & \\
\hline Age(years) & 1.007 & 0.996 & 0.991 & 1.006 & 0.977 & 1.017 & 1.016 \\
\hline \multicolumn{8}{|l|}{ Lifestyle } \\
\hline $\begin{array}{l}\text { Mental stress } \\
(\text { much }=1 \text {, others }=0)\end{array}$ & $2.699 * *$ & $2.626 * *$ & $3.538 * * *$ & 1.647 & 1.118 & $2.405^{*}$ & $3.539 * *$ \\
\hline $\begin{array}{l}\text { Nutritional balance } \\
\text { (enough= } 1 \text {, others }=0)\end{array}$ & 1.379 & 1.165 & 0.77 & 1.945 & 1.397 & $6.549^{* * *}$ & $4.698 * *$ \\
\hline $\begin{array}{l}\text { Breakfast } \\
(\text { almost everyday }=1)\end{array}$ & $2.340 *$ & $4.531 * * *$ & 0.908 & 1.780 & 2.337 & 1.646 & 2.489 \\
\hline $\begin{array}{l}\text { Physical exercise } \\
\text { (once or more per week }=1 \text { ) }\end{array}$ & 0.966 & 1.214 & 1.442 & 1.043 & 0.781 & 0.864 & 0.760 \\
\hline $\begin{array}{l}\text { Sleeping hours } \\
(7 \text { or more per } \text { day }=1 \text {, others }=0 \text { ) }\end{array}$ & 1.404 & $2.067^{*}$ & 1.249 & $1.946^{*}$ & 0.648 & 1.648 & 1.461 \\
\hline $\begin{array}{l}\text { Alcohol consumption } \\
\text { (once or less per day }=1 \text {, others }=0 \text { ) }\end{array}$ & 0.784 & 0.562 & 1.023 & 0.987 & 1.415 & 0.671 & 0.696 \\
\hline $\begin{array}{l}\text { Working hours per day } \\
(9 \text { or less per day }=1 \text {, others }=0)\end{array}$ & 0.982 & 1.085 & 1.298 & 0.808 & 0.28 & 0.767 & 0.845 \\
\hline $\begin{array}{l}\text { Cigarettes smoking } \\
\text { (quit or never } \text { smoked }=1, \text { smoking }=0 \text { ) }\end{array}$ & 0.962 & 1.223 & 0.992 & 0.939 & 0.622 & 1.297 & 1.039 \\
\hline
\end{tabular}

aSee Methods. ${ }^{*} p<0.05,{ }^{* *} p<0.01,{ }^{* * *} p<0.001$.

28 measures the overall mental health status. We believe that the group of individuals with higher GHQ-28 scores included those with poor mental health but did not necessarily suffer from depression. Moreover, the severe depression subscale of the GHQ-28 cannot detect mild depression in a normal population. Consequently, based on the scores obtained on these scales, we classified the subjects into four groups and identified the low-risk and high-risk groups with regard to the mental health status. The results of our study indicated that in the older age group, the HPI score was significantly correlated with the mental health status. This finding indicated that in the older age group, poor health practices have the potential to become multiple factors of poor mental health.

Furthermore, we observed that the anxiety-insomnia and social dysfunction subscales of the GHQ-28 significantly correlated with the HPI scores in the older age group. Ezoe et al. ${ }^{13)}$ reported that the scores for the total GHQ-28 and its three subscales (somatic symptoms, anxiety-insomnia, and social dysfunction) significantly decreased with the presence of a high total number of favorable lifestyle practices in both male and female workers. In the present study, similar trends were demonstrated between the different lifestyle factors and the GHQ- 28 scores, i.e., the more favorable the lifestyle, the lower were the scores of the male workers in the older age group. However, in the older age group, the number of significant subscales of the GHQ-28 was decreased. In contrast, no significant differences were observed in the younger age group. Since this study had a small sample size in comparison with that used in the previous research, the size might not yet be sufficiently large for obtaining statistically significant results between the groups. Clearly, these findings should be qualified by certain limitations imposed by the research methodology of the present investigation. The main limitation in the present study is that this survey involved participants from different workplaces who participated in the previous research. Moreover, the study of the workplace environment was not within the scope of this investigation. Therefore, inclusion of a greater variety of employees from different workplaces and further research in order to obtain more theoretical data of the lifestyle and mental health are necessary.

The results of the multiple logistic regression analysis indicated that four of the eight health practices, i.e., mental stress, nutritional balance, breakfast consumption, and sleeping hours, were significantly related to the total GHQ28 scores; the GHQ-28 subscale scores for somatic symptoms, anxiety-insomnia, and social dysfunction; the total SDS scores; or high-risk and low-risk groups for mental health status. In particular, eating habits and sleeping time 
appeared to be related to the mental health status; this finding is similar to the findings obtained in previous studies ${ }^{8,10,27)}$. In 1991, Simonsick ${ }^{25}$ ) suggested that dietary practices generally showed a negative association with mental health; those individuals who followed good dietary practices had a significantly higher risk of nervous breakdown than those who followed poor practices. Cooper et al..$^{28,29)}$ observed a strong relationship between long working hours and mental health as a source of stress among police and tax officers. Maruyama et al. ${ }^{27)}$ also reported that long working hours may be associated with poorer lifestyle, higher stress, and lower quality of life among middle-level managers. Moreover, Maruyama ${ }^{20)}$ also demonstrated that sufficient physical exercise predominantly resulted in fewer depressive symptoms in both males and females. Frederick ${ }^{24)}$, Farmer $^{30)}$, and Simonsick ${ }^{25)}$ further demonstrated that physical exercise reduces depressive symptoms and improves the mental health status. The results of the present study, however, indicated that these health practices had a limited relationship with the mental health status in factory workers. They also indicate that proper dietary habits can cause an improvement in the mental health status of persons who adopt the lifestyles, behavior patterns, perceived stressors, and stress reactions that are prevalent in the present time.

Berkman and Breslow ${ }^{31)}$ and Kusaka et al..$^{7)}$ proposed that a poor lifestyle results in numerous physical health problems. Furthermore, Maruyama et al. ${ }^{12)}$ reported that lifestyles affect the mental health status. Pratt ${ }^{32}$, Williams and Wechler ${ }^{33)}$, and Matarazzo ${ }^{34)}$ observed that a poorer quality of health practices was related to a higher subjective level of health and to an increased number of health problems. For example, a lack or excess of sleep or minimal physical exercise might lead to symptoms of depression. These results also provided highly suggestive evidence that the lifestyle followed in the face of stress may reduce the risk of stress-related illnesses. Previously, the GHQ-28 and SDS were used for the evaluation of stress reactions ${ }^{22,35)}$, and both these questionnaires demonstrated the interaction between subjective and physiological stress. In this research, we also affirmed that the concurrent assessment of the mental health status by using both the GHQ-28 and SDS questionnaires is beneficial for classifying normal subjects into high-risk or low-risk groups as a stress-related illness risk factor. However, none of the groups whose characteristics were considered to be unhealthy (high GHQ-28 or SDS scores) exhibited significant differences in the health practices followed. This may be due to a small sample size. Hence, larger study populations are required for further studies to clarify these trends.

In summary, our study on factory workers suggests that good health practices are closely associated with good mental health. However, the methodological limitations of the current study should be considered while assessing the results. Since this was a cross-sectional study, its limitations should be considered while interpreting the findings. Thus, further prospective studies are required to clarify the theoretical causal mechanism of factors that modify the lifestyle-mental health relationship. We plan to test larger and more varied populations and increase the scope of the study. Based on the current results, it may be stated that intervention programs aimed at changing lifestyles to improve physical health and prevent ischemic heart disease and cancer may also help in improving the mental health of factory workers.

\section{References}

1) Morimoto K, Miura K, Kaneko T, Iijima K, Koizumi A (1984) Health situation and chromosome alterations: Sister chromatid exchange frequency in lymphocyte from passive smokers and patients with hereditary diseases. In: Sister Chromatid Exchanges: Genetic Toxicology and Human Studies, Tice R, Hollaender A, Lambert B and Morimoto K (Eds.), 80112, Plenum, New York.

2) Ezoe S, Araki S, Ono Y, Kawakami N, Murata K (1994) Assessment of personality traits and psychiatric symptoms in a computer manufacturing plant in Japan. Am J Ind Med 25, 187-96.

3) Belloc NB, Breslow L (1972) Relationship of physical health status and health practices. Prev Med 1, 409-21.

4) Belloc NB (1973) Relationship of health practices and mortality. Prev Med 2, 67-81.

5) Berkman LF, Breslow L (1983) Health and Ways of Living. Oxford Univ. Press, New York.

6) Morimoto K (1990) Life-style and genetic factors that determine the susceptibility to production of chromosome damage. In: Chromosomal Aberrations: Basic and Applied Aspects, Obe G and Natarajan AT (Eds.), 287-301, SpringerVerlag, Berlin.

7) Kusaka Y, Kondou H, Morimoto K (1992) Healthy lifestyles are associated with higher natural killer cell activity. Prev Med 21, 602-15.

8) Hagiwara A, Morimoto K (1991) Personal health practice and attitudes toward nonsmokers' legal rights in Japan. Soc Sci Med 33, 717-21.

9) Goldberg DP (1972) The Detection of Psychiatric Illness by Questionnaire. Maudsley Monograph No. 21, Oxford Univ. Press, London.

10) McDowell I, Newell C (1987) Measuring Health, Oxford Univ. Press, New York.

11) Barrett J, Hurst MW, Discala C (1978) Prevalence of depression over a 12-month period in a nonpatient population. Arch Gen Psychiatry 3, 741-4.

12) Maruyama S, Sato H, Morimoto K (1991) Relationship 
between work-life satisfaction, health practice and primary symptoms/problems. Nippon Eiseigaku Zasshi 45, 1082-94.

13) Ezoe S, Morimoto K (1994) Behavioral lifestyle and mental health status of Japanese factory workers. Prev Med 23, 98 105.

14) Zung WWK (1965) A self-rating depression scale. Arch Gen Psychiatry 12, 63-70.

15) Zung WWK (1986) Zung self-rating depression scale and depression status inventory. In: Assessment of Depression, Sartorius N and Ban TA (Eds.), 221-31, Springer-Verlag, Berlin.

16) Chida F, Okayama A, Nishi N, Sakai A (2004) Factor analysis of Zung Scale scores in a Japanese general population. Psychiatry Clin Neurosci 58, 420-6.

17) Fukuda K, Kobayashi S (1973) A study on a self-rating depression scale (author's translation). Seishin Shinkeigaku Zasshi 75, 673-9 (in Japanese).

18) Sarai K (1979) Epidemiology of depression. Jpn Psychiat Neurol 82, 777-84 (in Japanese).

19) Morimoto K (1994) Theoretical and practical perspectives of occupational stress research-towards a new theory of mental health based on individual variations. Job Stress Res 1, 49-63 (in Japanese).

20) Maruyama S, Morimoto K (1997) The effects of lifestyle and type A behavior on the life-stress process. Environ Health Prev Med 2, 28-34.

21) Middleton W, Raphael B, Burnett P, Martinek N (1997) Psychological distress and bereavement. J Nerv Ment Dis 185, 447-53.

22) Toda M, Makino H, Kobayashi H, Nagasawa S, Kitamura K, Morimoto K (2004) Medical assessment of the health effects on short leisure trips. Arch Environ Health 59, 717-24.

23) Kitamura T, Shima S, Sugawara M, Toda MA(1994) Temporal variation of validity of self-rating questionnaires: repeated use of the General Health Questionnaire and Zung's Selfrating Depression Scale among women during antenatal and postnatal periods. Acta Psychiatr Scand 90, 446-50.

24) Fredrick T, Frerichs RR, Clark VA (1988) Personal health habits and symptoms of depression at the community level. Prev Med 17, 173-82.

25) Simonsick EM (1991) Personal health habits and mental health in a national probability sample. Am J Prev Med 7, 425-37.

26) Wetzler HP, Ursano RJ (1988) A positive association between physical health practices and psychological well-being. J Nerv Ment Dis 176, 280-3.

27) Maruyama S, Morimoto K (1996) Effects of long workhours on life-style, stress and quality of life among intermediate Japanese managers. Scand J Work Environ Health 22, 353-9.

28) Cooper CL, Davidson MJ, Robinson P (1982) Stress in the police service. J Occup Med 24, 30-6.

29) Cooper CL, Roden J (1985) Mental health and satisfaction among tax officers. Soc Sci Med 21, 747-51.

30) Farmer ME, Locke BZ, Moscicki EK, Dannenberg AL, Larson DB, Radloff LS (1988) Physical activity and depressive symptoms. The NHANES I epidemiologic follow-up study. Am J Epidemiol 128, 1340-51.

31) Berkman LF, Breslow L (1983) Health and Ways of Living: The Alameda County Study, Oxford Univ. Press, New York.

32) Pratt L (1971) The relationship of socioeconomic status to health. Am J Public Health 61, 281-91.

33) Williams AF, Wechsler H (1972) Interrelationship of preventive actions in health and other areas. Health Service Reports 87, 969-76.

34) Matarazzo J (1982) Behavioral health's challenge to academic, scientific, and professional psychology. Am Psychol 37, 114.

35) Toda M, Morimoto K, Nagasawa S, Kitamura K (2006) Change in salivary physiological stress markers by spa bathing. Biomed Res 27, 11-4. 